\title{
ORIGINAL
}

\section{Measurement of hemodynamics immediately after vaginal delivery in healthy pregnant women by electrical cardiometry}

\author{
Atsuko Yoshida', Takashi Kaji ${ }^{1}$, Hirotsugu Yamada ${ }^{2,3}$, Naoto Yonetani ${ }^{1}$, Eishi Sogawa', Masami Yamao ${ }^{3}$, \\ Kazuhisa Maeda ${ }^{1,4}$, Masataka Sata ${ }^{5}$, and Minoru Irahara ${ }^{1}$

\begin{abstract}
${ }^{1}$ Department of Obstetrics and Gynecology, Institute of Health Biosciences, the University of Tokushima Graduate School, Tokushima, ${ }^{2}$ Department of Community Medicine for Cardiology, Tokushima University Graduate School of Medicine, Tokushima, Japan, ${ }^{3}$ Ultrasound Examination Center, Tokushima University Hospital, Tokushima, Japan, ${ }^{4}$ Perinatal Medical Center, Shikoku Medical Center for Children and Adults, Japan, ${ }^{5}$ Department of Cardiovascular Medicine, Tokushima University Graduate School of Medicine, Tokushima, Japan
\end{abstract}

\begin{abstract}
Few reports have focused on hemodynamics around delivery in pregnant women because of the difficulty of continuous and noninvasive measurement. Electrical cardiometry allows noninvasive continuous monitoring of hemodynamics and has recently been used in non-pregnant subjects. We compared the use of electrical cardiometry versus transthoracic echocardiography in healthy pregnant women and evaluated hemodynamics immediately after vaginal delivery. In Study 1, electrical cardiometry and transthoracic echocardiography were used to measure cardiac output in 20 pregnant women with threatened premature delivery. A significant correlation was found between the two methods, with electrical cardiometry showing the higher cardiac output. In Study 2 , heart rate, stroke volume, and cardiac output were continuously measured in 15 women during vaginal delivery up to $2 \mathrm{~h}$ postpartum. Cardiac output increased markedly because of an increased heart rate and stroke volume at the time of newborn delivery. The heart rate then immediately returned to baseline, while cardiac output remained elevated for at least $2 \mathrm{~h}$ after delivery because of a sustained high stroke volume. Electrical cardiometry was as readily available as transthoracic echocardiography for evaluating hemodynamics and allowed for continuous measurement during labor. High intrapartum cardiac output was sustained for at least $2 \mathrm{~h}$ after vaginal delivery. J. Med. Invest. 66 :75-80, February, 2019
\end{abstract}

Keywords : Electrical cardiometry, Pregnancy, Parturition, Hemodynamics, Vaginal delivery

\section{INTRODUCTION}

Pregnancy has a major impact on the cardiovascular system of pregnant women. Pregnancy-induced changes are most dramatic during labor $(1,2)$. The incidence of cardiac disease in pregnant women is increasing, and peripartum cardiomyopathy (PPCM), a severe disease that causes heart failure in pregnant women, has become widely recognized (3). Thus, the importance of assessing cardiac function in pregnant women has considerably increased.

No generally accepted theory of how circulatory dynamics change during and immediately after delivery has been established because evaluation methods are limited. Transthoracic echocardiography (TTE) and the thermodilution method using a pulmonary artery catheter have been used to evaluate circulation dynamics (4). Thermodilution is the clinical gold standard for measuring cardiac output (CO), but this method is invasive and is now rarely performed (5). In contrast, TTE is widely used in pregnant women because of its noninvasiveness (6) ; however, continuous monitoring is difficult.

Electrical cardiometry (EC) is a recently developed technique for monitoring hemodynamics (7). EC is used to measure the

Received for publication June 8, 2018 ; accepted August 24, 2018.

Address correspondence and reprint requests to Atsuko Yoshida, Department of Obstetrics and Gynecology, Institute of Health Biosciences, the University of Tokushima Graduate School, 3-8-15 Kuramoto-cho, Tokushima 770-8503, Japan and Fax : +81-88-631-2630. stroke volume (SV) and thus monitor changes in red blood cells flowing through the aorta ; this involves attaching four electrocardiographic electrodes to the body to assess changes in conductivity. EC is noninvasive and simple and allows for continuous monitoring of cardiac function. Thus, it has been used in patients in the intensive care unit, patients undergoing dialysis, and newborns. However, few reports have described the use of EC in pregnant women. The present study was performed to verify the utility of EC in pregnant women by comparison with TTE. We also examined patients' hemodynamics immediately after vaginal delivery using EC.

\section{PATIENTS AND METHODS}

Patients

We performed two studies. Study 1 was a comparative study of TTE and EC in pregnant women, and Study 2 was an observational study in which patients' hemodynamics were continuously monitored during and after vaginal delivery. Our institutional review board approved these studies, and written informed consent was obtained from each patient enrolled in the studies.

In Study 1 (June 2014-December 2014), we enrolled 20 patients in our hospital who had been diagnosed with threatened premature delivery (Table 1 ). 
Table 1. Biometric data

\begin{tabular}{ccc}
\hline & Study 1 & Study 2 \\
\hline Age, years & $30.4 \pm 5.7$ & $33.9 \pm 4.3$ \\
Height, cm & $161.2 \pm 21.1$ & $156.9 \pm 6.2$ \\
Weight, kg & $61.9 \pm 10.5$ & $66.0 \pm 9.9$ \\
Heart rate, beats/min & $87.6 \pm 34.1$ & - \\
Systolic blood pressure, $\mathrm{mmHg}$ & $107.6 \pm 43.4$ & $116.1 \pm 10.2^{\star}$ \\
Diastolic blood pressure, mmHg & $61.5 \pm 31.5$ & $69.1 \pm 10.5^{\star}$ \\
Gestational age, weeks & $30.1 \pm 8.6$ & $39.0 \pm 1.0$ \\
Rate of oxytocin use, \% & - & 47.7 \\
Delivery time, hours & - & $5.9 \pm 4.1$ \\
Amount of bleeding during & - & $259.4 \pm 94.6$ \\
delivery, g & - & $3133.6 \pm 339.8$ \\
Birth weight, g &
\end{tabular}

Data are presented as mean \pm standard deviation.

Physical data correspond to the time of delivery (except for the rate of oxytocin use, which is presented as the percentage of the total number of patients).

*Immediately after delivery.

The patients had received intravenous infusions of ritodrine hydrochloride for tocolysis. Patients with complications such as hypertensive disorders of pregnancy, cardiac malformations, or severe anemia were excluded. All patients were in sinus rhythm and had normal cardiac function.

In Study 2 (June 2015-June 2016), we enrolled 15 pregnant women without complications (Table 1). The patients underwent labor at term that was either induced by (or augmented with) oxytocin or spontaneous. The infusion volume was limited to only the amount necessary to induce/augment labor, or it was not administered. Patients were excluded if they required additional treatment, such as infusion for atonic bleeding or magnesium sulfate for hypertensive disorders of pregnancy. Oxytocin was initiated concurrently with the start of induction or weak labor pain. Our hospital-based oxytocin protocol was used for all patients ; this started with $1.7 \mathrm{mU} / \mathrm{min}$ and then increased by $1.7 \mathrm{mU}$ every 30 min until regular uterine contractions occurred. The maximum dose of oxytocin was $20 \mathrm{mU}$, with no limit to the length of time a patient could remain at $20 \mathrm{mU}$. When the oxytocin infusion was continued after umbilical cord clamping, it was administered at the same dose.

\section{Echocardiography}

All measurements were obtained with the patient resting in the left lateral position to optimize TTE imaging. An experienced cardiac sonographer starting monitoring CO with both TTE and EC within 10 min after the start of TTE. TTE was performed using an echocardiographic Doppler system (Vivid E9 ; GE Healthcare, Chalfont St. Giles, UK). Measurements and recordings were obtained according to the recommendations of the American Society of Echocardiography (8). CO was determined by Doppler echocardiographic measurement of the left ventricular outflow tract (LVOT) velocity combined with the cross-sectional area with measurement of the LVOT diameter. The flow velocity was measured with pulsed-wave Doppler echocardiography by placing a transducer on the apex. An average of 8 to 10 consecutive beats was used for each determination. The diameter of the LVOT was measured during systole from a cross-sectional echocardiographic view (parasternal long-axis plane). Diameters from five consecutive beats were averaged.

The LVOT cross-sectional area was calculated from the equation $(\mathrm{D} / 2)^{2} \pi$, where D is the mean LVOT diameter. Heart rate (HR) was determined from the $\mathrm{R}-\mathrm{R}$ interval of a simultaneously recorded electrocardiogram. $\mathrm{CO}$ was then calculated according to the following formula : $\mathrm{CO}(\mathrm{ml} / \mathrm{min})=$ stroke volume $(\mathrm{SV})(\mathrm{ml}) \times \mathrm{HR}(/ \mathrm{min})$, where SV $(\mathrm{ml})$ is calculated as time velocity integral $(\mathrm{cm}) \times$ crosssectional area $\left(\mathrm{cm}^{2}\right)$. End-systolic and end-diastolic dimensions were measured from standard left ventricular M-mode echocardiographs. Based on these algorithms, $\mathrm{CO}$ was measured by TTE (TTE-CO).

\section{Electrical cardiometry}

$\mathrm{EC}$ is based on a bioimpedance technique that is used to measure changes in transthoracic impedance during cardiac ejection caused by volumetric changes of the aorta. We used an AESCULON Mini (Osypka Medical GmbH, Berlin, Germany) for EC. Four disposable electrocardiographic electrodes were attached at the neck and the inferior aspect of the thorax at the level of the xiphoid process to record the changing impedance over that area of the thorax. A highfrequency $(50-\mathrm{kHz})$, low-amperage $(2-\mathrm{mA})$ alternating electrical current of constant amplitude was emitted via a pair of surface electrodes across the left thorax. The inner electrodes acted as sensing electrodes to register electrical resistance changes caused by application of the current and recorded the thoracic electrical bioimpedance $(Z)$.

$\mathrm{Z}=$ sensed voltage

applied current

Verification of the correct signal quality was accomplished by visualizing the electrocardiogram, the impedance waveform, and its first derivative. The maximum rate of change in the thoracic electrical bioimpedance over that area was interpreted as the ohmic equivalent of the mean blood flow velocity in the ascending aorta. SV ( $\mathrm{ml})$ and $\mathrm{CO}(\mathrm{L} / \mathrm{min})$ were then calculated using the following equations :

$\mathrm{SV}=\mathrm{V}_{\mathrm{EPT}} \times V \times \mathrm{LVET}$

$\mathrm{CO}=\mathrm{SV} \times \mathrm{HR} / 100$,

where $\mathrm{V}_{\text {ЕPT }}(\mathrm{ml})$ is the volume of electrically participating tissue derived from the body mass and height, $v(/ \mathrm{s})$ is the ohmic equivalent of the mean aortic blood flow velocity during left ventricular ejection, and LVET (s) is the left ventricular ejection time. The impedance recordings and $\mathrm{CO}$ and SV calculations were obtained using the AESCULON Mini, incorporating an algorithm that transforms the ohmic equivalent of mean aortic blood flow acceleration into an equivalent of mean aortic blood flow velocity : $v=\left[\frac{(d Z(t) / d t) \min }{Z_{0}}\right]_{\mathrm{n}}$

where $[(d Z(t) / d t) \min ]$ is the maximum rate of change of the thoracic electrical bioimpedance during systole and $Z_{0}$ is the base impedance (average value over 10 cardiac cycles). Based on these algorithms, EC was used to measure CO (EC-CO).

\section{Protocol for Study 1}

Each TTE measurement was paired with the EC measurement that most closely followed it in time, and only TTE and EC data pairs measured within $20 \mathrm{~s}$ of each other were included. This resulted in multiple data pairs for each patient ; these pairs were then averaged to obtain one value per patient for each variable. The average values were used for statistical analysis. The closest EC measurement following the velocity-time integral image was chosen because EC data were acquired as the moving average of the previous 10 heartbeats, whereas TTE data reflect one velocity-time integral image obtained at a single instant in time. This explicit pairing of EC and TTE measurements was initially performed to compare all paired EC and TTE measurement sets individually for a given patient. However, we later decided to report one average value per patient for each technology.

\section{Protocol for Study 2}

We set up the AESCULON Mini for each patient at the begin- 
ning of labor to measure hemodynamics. HR, SV, and CO were measured in beat-to-beat mode. All patients were maintained in the dorsal lithotomy position during delivery and in the supine position after delivery. The measurements were obtained at predefined time points : labor onset, newborn delivery, and at 10, 15, 20, 30, 60, 90, and $120 \mathrm{~min}$ after delivery. Measurement at labor onset was performed during an intermittent period during uterine contractions. Hemodynamic data were stored in the AESCULON Mini monitor and downloaded as text files.

\section{Statistical analysis}

For Study 1, the correlation between TTE-CO and EC-CO values was evaluated by calculating Pearson's correlation coefficient $r$ and applying a linear regression model of TTE-CO on EC-CO. The spread of the slope and the ordinate of this relationship were expressed as a standard error. The correlation coefficient was then calculated to allow comparison of our results with those of other studies. The differences between the paired $\mathrm{CO}$ values were also plotted against the average $\mathrm{CO}$ values of both measurements. This statistical method was recommended by Bland-Altman for evaluation studies. To investigate the relationship between the measurement error and the true value, the values were plotted as the difference between the methods and their means. The limits of agreement were estimated by calculating the mean difference (bias) and standard deviation (SD). The upper and lower limits were defined as the bias $\pm 2 \mathrm{SD}$, the range in which $95 \%$ of the differences between the methods were expected to occur. A $P$ value of $<0.05$ was considered to indicate statistical significance.

For Study 2, the Wilk-Shapiro test was used to assess the normality of the distribution of data. All continuous, normally distributed measurements are presented as mean \pm SD. Categorical data are reported as median with interquartile range. Comparisons of hemodynamic variables from labor onset and newborn delivery to the other predefined time points were performed using analysis of variance with repeated measures. All data were analyzed using EZR (Saitama Medical Center, Jichi Medical University, Saitama, Japan), a graphic user interface for R (The R Foundation for Statistical Computing, Vienna, Austria); EZR is a modified version of $\mathrm{R}$ Commander designed to add statistical functions frequently used in biostatistics. The significance level was set $P<$ 0.05 .

\section{RESULTS}

Study 1 : Relationships between TTE-CO and EC-CO

In total, 20 patients were enrolled in Study 1. No patients were removed from the analysis (i.e., TTE-CO andEC-CO values were obtained for all patients). No serious complications occurred during the study. The pooled EC-CO was 3.68 to $8.10 \mathrm{~L} / \mathrm{min}$, and TTE-CO was 3.35 to $7.10 \mathrm{~L} / \mathrm{min}$. Figure 1 shows a scatter plot of the data with the linear regression line.

A significant correlation was found between the two methods $(P<0.001)$, with $r=0.79$. EC-CO was linearly related to TTE-CO, with a y-intercept of 1.19 and a line slope of 0.85 (i.e., the EC-CO values were higher than TTE-CO values). Bland-Altman analysis data are shown in Figure 2.

The mean difference (bias) between EC-CO and TTE-CO was $0.36 \mathrm{~L} / \mathrm{min}(\mathrm{SD}, 0.67 \mathrm{~L} / \mathrm{min})$. The limits of agreement were defined as the mean difference $\pm 2 \mathrm{SD}$. The lower and upper limits of agreement for the comparison of EC-CO with TTE-CO were -0.98 and $1.7 \mathrm{~L} / \mathrm{min}$, respectively. The rate of relative errors within $\pm 20 \%$ was $95 \%$ of all measurements.

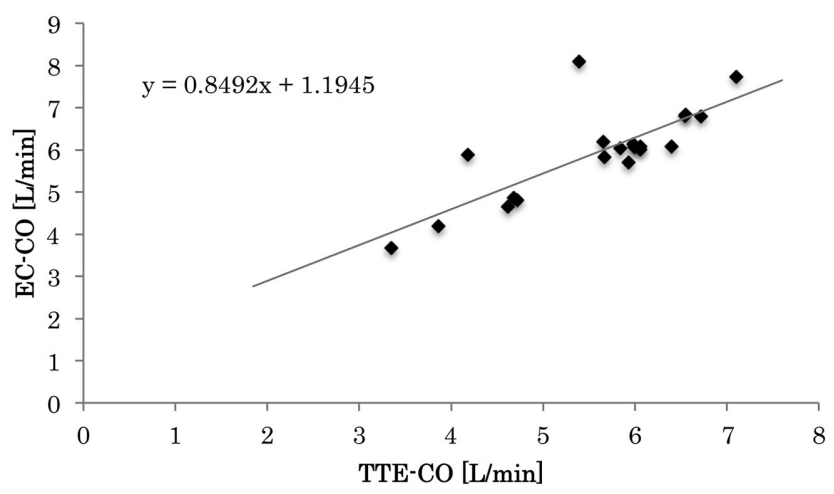

Fig. 1 Scatter plot comparing cardiac output. This graph denotes the linear regression line. TTE-CO, cardiac output as measured by transthoracic echocardiography ; EC-CO, cardiac output as measured by electrical cardiometry

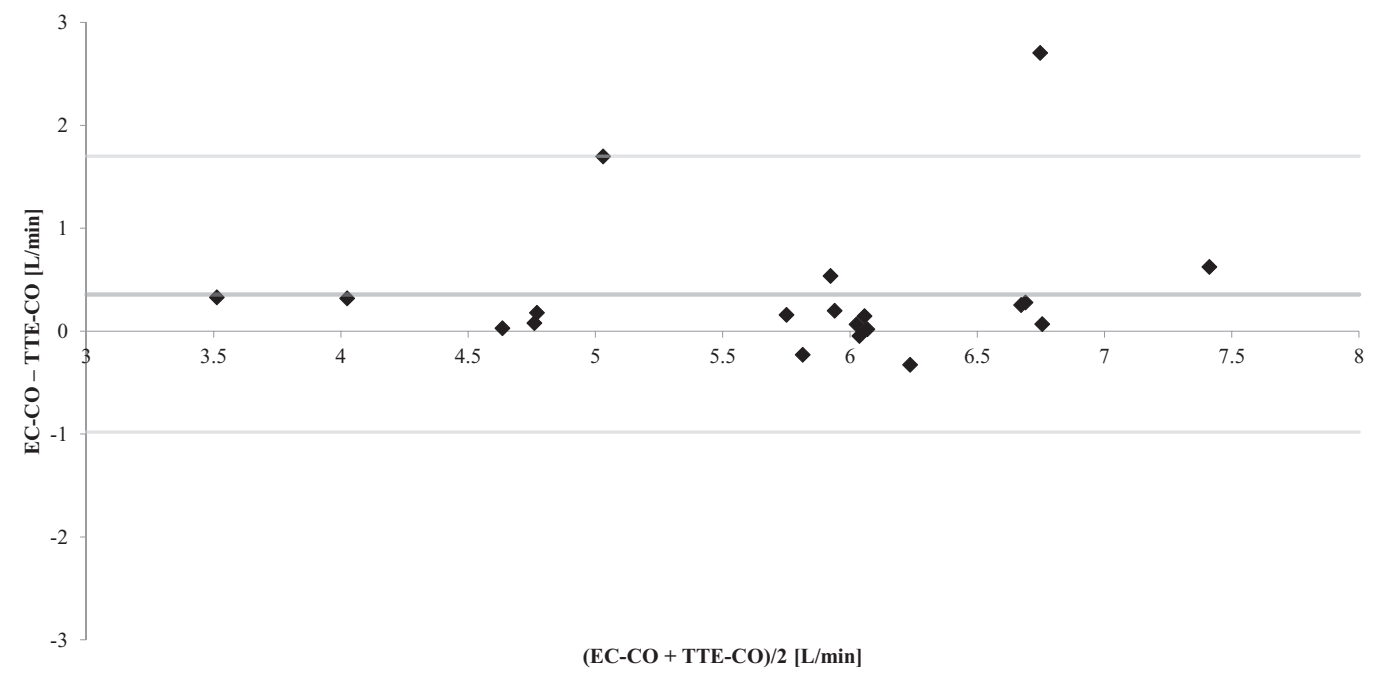

Fig. 2 Bland-Altman plot. Bias plot of the difference in cardiac output as measured by EC and TTE compared with the mean of the two results. The mean difference in EC-TTE was $0.36 \mathrm{~L} / \mathrm{min}$ (standard deviation, $0.67 \mathrm{~L} / \mathrm{min}$ ). The lower and upper limits of agreement were $-0.98 \mathrm{and} 1.7 \mathrm{~L} / \mathrm{min}$, respectively. TTE-CO, cardiac output as measured by transthoracic echocardiography ; EC-CO, cardiac output as measured by electrical cardiometry 
Study 2 : Hemodynamic changes during and after vaginal delivery

Hemodynamic variables measured at the predefined time points are shown in Figure 3.

A significant increase in $\mathrm{HR}, \mathrm{SV}$, and $\mathrm{CO}$ occurred at the time of newborn delivery compared with the baseline value measured at the onset of labor. HR, SV, and CO increased by $32.9 \%, 50.5 \%$, and 95.0\% from baseline, respectively. At $10 \mathrm{~min}$ after delivery, HR fell to that observed at labor onset and remained constant. In contrast, SV and $\mathrm{CO}$ at 10 min after delivery decreased from that seen during newborn delivery, but remained higher than that at labor onset. SV and CO remained stable until 120 min after delivery, with values $20.4 \%$ and $22.5 \%$ higher than those at labor onset, respectively.

\section{DISCUSSION}

We confirmed that EC can be used to evaluate the hemodynamics of pregnant women, with findings similar to those of TTE. We also found that a high-CO state persisted for at least $2 \mathrm{~h}$ after vaginal delivery because of the sustained high SV.

The thermodilution method using a pulmonary artery catheter is the accepted gold standard for evaluation of $\mathrm{CO}$, but this technique is invasive and risky. Hence, TTE is now generally used because it is simple and noninvasive. Indeed, TTE is reportedly useful in pregnant women with the same accuracy as thermodilution (9). A disadvantage of TTE is that the technique needs to be learned. Further, the same posture must be maintained for a prolonged time, which is difficult for pregnant patients. Finally, TTE cannot be used for continuous measurement. Therefore, there are some limits to using TTE for evaluation of hemodynamics in pregnant women, especially during labor and delivery.

In contrast to thermodilution and TTE, EC uses the increase in conductivity from the change in orientation of red blood cells to determine the velocity of blood flow, allowing SV and CO measurement (10). EC is easy to use, and there is no restriction caused by the position or physique of the patient. Previous studies have demonstrated that $\mathrm{EC}$ is a reliable technique for measuring $\mathrm{CO}$ compared with well-established methods such as the direct Fick's oxygen principle, thermodilution, or transesophageal echocardiography $(11,12)$. In obstetrics, $\mathrm{EC}$ is also used to measure the a

[/min]

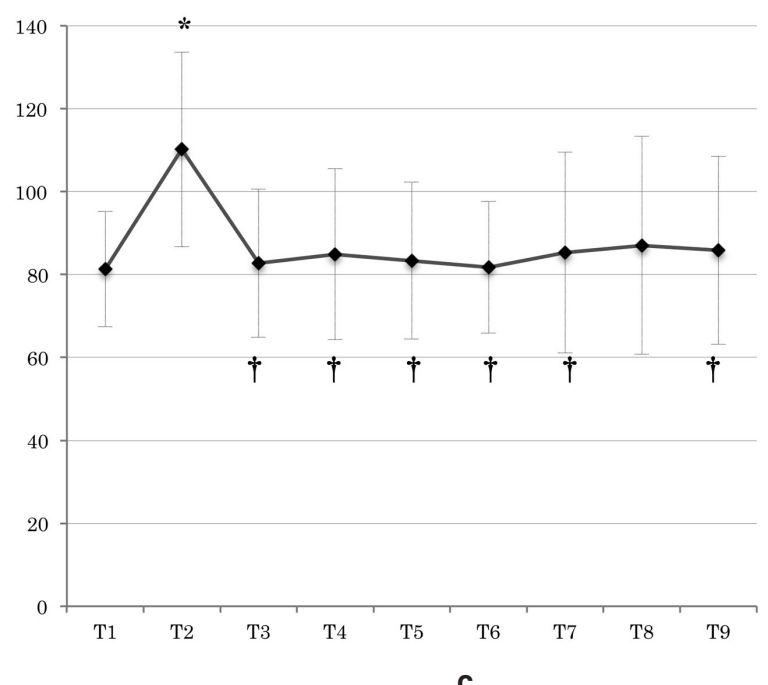

b

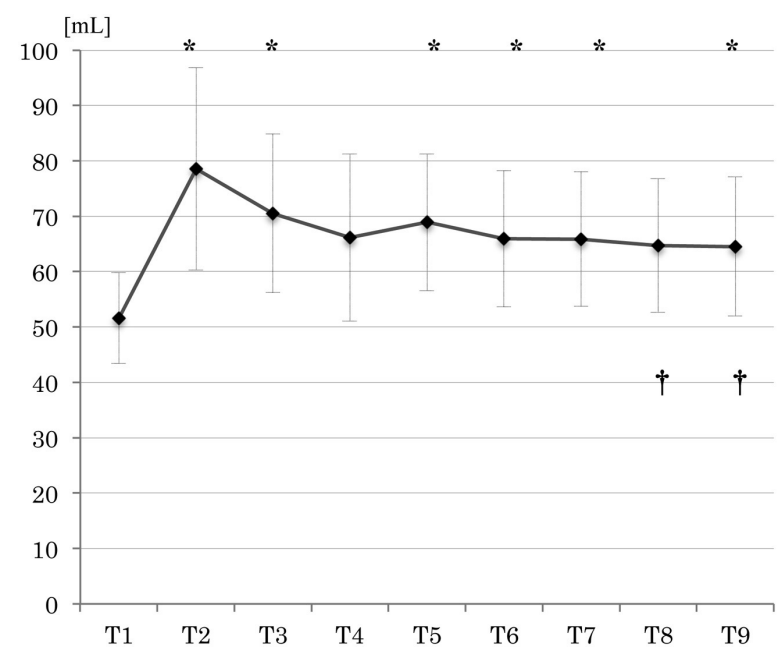

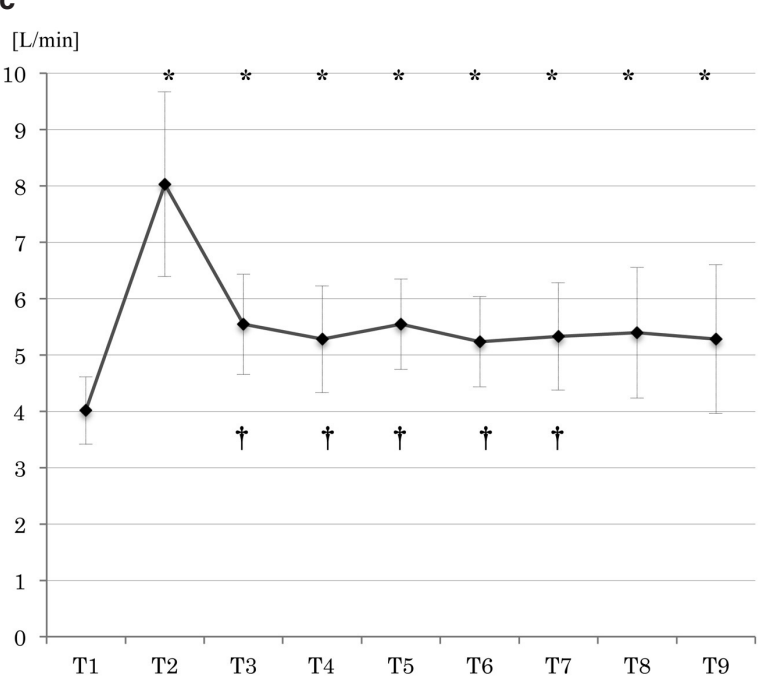

Fig. 3 Circulation fluctuation around the time of vaginal delivery. (a) Heart rate. (b) Stroke volume. (c) Cardiac output. Data are presented as mean (95\% confidence limit). The marked groups were considered statistically significant $(P<0.05)$ in comparison with *labor onset and †newborn delivery. Time points : baseline measurement at labor onset (T1), newborn delivery (T2), and after delivery at $10 \mathrm{~min}$ (T3), $15 \mathrm{~min}$ (T4), $20 \mathrm{~min}$ (T5), $30 \mathrm{~min}$ (T6), $60 \mathrm{~min}$ (T7), $90 \mathrm{~min}$ (T8), and $120 \min$ (T9) 
hemodynamic changes associated with cesarean delivery and oxytocin administration $(13,14)$. Importantly, EC can provide continuous and noninvasive measurement without the need for the operator to undergo a learning curve.

In the present study, EC showed a trend toward higher values than TTE, although we confirmed the compatibility of the values measured by the two methods. Grollmuss and Gonzalez (15) also found that EC produced higher values than TTE. Moreover, TTE has been shown to underestimate CO compared with magnetic resonance imaging, which is considered the most accurate noninvasive method for evaluating cardiac function, as in our study (16-18). Our data support that EC is a useful technique, particularly for continuous evaluation of hemodynamics.

Because EC has been proven feasible for pregnant women as well as TTE, we attempted to evaluate the hemodynamics around the time of vaginal delivery using EC. Although dramatic hemodynamic changes are known to occur during labor, few reports have focused on detailed hemodynamic measurements around the delivery period, especially immediately after delivery. Robson et al. (19) reported that $\mathrm{CO}$ increased for at least $1 \mathrm{~h}$ after vaginal delivery because of increased SV, although the HR did not change. By $24 \mathrm{~h}$ after delivery, there were no significant increases in CO, HR, or SV. However, San-Frutos et al. (20) reported that CO and SV continued to decline up to $48 \mathrm{~h}$ after delivery and then increased compared with these values during early pregnancy. Although both studies performed evaluations at $48 \mathrm{~h}$ after delivery, they did not evaluate changes around and immediately after delivery, and their recordings were not continuous. Hemodynamic parameters readily vary in accordance with factors such as the patient's posture, bleeding, pain, and mental state. Therefore, continuous measurement as in the present study is useful for elucidating the maternal circulation variations that occur during delivery.

It is easy to imagine that hemodynamic parameters change markedly around vaginal delivery, but whether this actually occurs remains unknown. Thus, it is important to measure the physiological hemodynamics of pregnant women. In the present study, HR was high up to the time of delivery and then rapidly decreased to the levels observed at the start of labor. The perception of pain and activation of sympathetic nerves are diminished by labor, which may explain the HR fluctuation. SV was markedly higher at delivery and then declined, but remained elevated at $2 \mathrm{~h}$ postpartum compared with that at the onset of labor ; thus, high $\mathrm{CO}$ also persisted for at least $2 \mathrm{~h}$ after vaginal delivery. This phenomenon may be caused by a temporary increase in the circulating blood volume, such as an autologous blood transfusion from the uterus and/or release of inferior vena cava compression associated with uterine contractions.

Heart load is an aggravating factor associated with heart disease and can induce heart failure such as that caused by PPCM. PPCM often develops during labor to around the time of delivery $(21,22)$, and the resulting heart failure can be serious, sometimes even leading to death. The main pathological condition observed in PPCM is congestion and low CO. Based on the findings from the present study, healthy pregnant women have high $\mathrm{CO}$ with increasing HR and SV at the time of delivery, and the high-CO state continues due to high SV. Thus, the heart failure associated with PPCM may be induced by decreased contractility at a time when it should inherently respond by increasing CO. EC may be useful for monitoring circulatory changes in high-risk patients with PPCM.

The present study has three limitations. First, we included only healthy pregnant women. High-risk patients with complications were excluded, such as patients with multiple pregnancies, heart disease, or a hypertensive disorder of pregnancy. The circulatory dynamics during puerperium in these patients may be different from those of normal pregnant women. Hemodynamic monitoring for such high-risk patients is specifically required from the view- point of the risk of complications, including heart failure and PPCM. Multiple reports have described the use of EC in nonpregnant patients with these diseases $(23,24)$. EC has been validated with acceptable reliability in ill adults $(25,26)$. Hence, we suggest that EC can also be used to evaluate hemodynamics in pregnant women with these complications. Second, the patients in Study 1 were at preterm gestation and those in Study 2 were at term gestation. Additionally, ritodrine hydrochloride was used in Study 1, but it was not used in Study 2. Impedance might be differentially impacted at different gestational ages and with the use of different drugs. Therefore, the correlation between EC and TTE verified in Study 1 cannot necessarily be generalized to Study 2 . Finally, in Study 2, we only studied pregnant women who went into labor without anesthesia and in the Farrar position. Because patient positioning and anesthesia affect circulation dynamics, our findings may not apply to delivery with patients in other positions and under anesthesia.

In summary, we confirmed that EC allows for evaluation of hemodynamic parameters with clinically acceptable accuracy for pregnant women because of its compatibility with TTE. We also succeeded in evaluating hemodynamics using EC around the time of vaginal delivery. We confirmed that $\mathrm{CO}$ remained elevated during labor and immediately after vaginal delivery. Because some pregnant women may have cardiovascular complications and poor cardiac function, particular attention should be paid to their postpartum circulatory dynamics. EC can provide constant monitoring of cardiac function over time ; therefore, we suggest that EC may support safe delivery management. For high-risk cases, such as women with hypertensive disorders of pregnancy or cardiac complications, more careful management is necessary, and hemodynamic monitoring with EC may provide important information.

\section{CONFLICTS OF INTEREST}

Atsuko Yoshida declares no conflicts of interest. Takashi Kaji declares no conflicts of interest. Hirotsugu Yamada declares no conflicts of interest. Naoto Yonetani declares no conflicts of interest. Eishi Sogawa declares no conflicts of interest. Masami Yamao declares no conflicts of interest. Kazuhisa Maeda declares no conflicts of interest. Masataka Sata declares no conflicts of interest. Minoru Irahara declares no conflicts of interest.

\section{FUNDING}

This research did not receive any specific grant from funding agencies in the public, commercial, or not-for-profit sectors.

\section{ACKNOWLEDGEMENT}

We thank Angela Morben, DVM, ELS, from Edanz Group (www. edanzediting.com/ac), for editing a draft of this manuscript.

\section{REFERENCES}

1. Cornette J, Roos-Hesselink J : Normal cardiovascular adaptation to pregnancy. In : Stergiopoulos K, ed. Evidence-based Cardiology Consult. Springer, London, 2014, pp.423-432

2. Melchiorre K, Sharma R, Thilaganathan B : Cardiac structure and function in normal pregnancy. Curr Opin Obstet Gynecol $24: 413-421,2012$ 
3. Pearson GD, Veille JC, Rahimtoola S, Hsia J, Oakley CM, Hosenpud JD, Ansari A, Baughman KL : Peripartum cardiomyopathy : national heart, lung, and blood institute and office of rare diseases (national institutes of health) workshop recommendations and review. JAMA $283: 1183-1188,2000$

4. Moshkovitz Y, Kaluski E, Milo O, Vered Z, Cotter G : Recent developments in cardiac output determination by bioimpedance : comparison with invasive cardiac output and potential cardiovascular applications. Curr Opin Cardiol 19 : 229-237, 2004

5. Ivanov R, Allen J, Calvin JE : The incidence of major morbidity in critically ill patients managed with pulmonary artery catheters : a meta-analysis. Crit Care Med 28 : 615-619, 2000

6. Katz R, Karliner JS, Resnik R: Effects of a natural volume overload state (pregnancy) on left ventricular performance in normal human subjects. Circulation $58: 434-441,1978$

7. Zoremba N, Bickenbach J, Krauss B, Rossaint R, Kuhlen R, Schälte G : Comparison of electrical velocimetry and thermodilution techniques for the measurement of cardiac output. Acta Anaesthesiol Scand $51: 1314-1319,2007$

8. Lang RM, Badano LP, Mor-Avi V, Afilalo J, Armstrong A, Ernande L, Flachskampf FA, Foster E, Goldstein SA, Kuznetsova T, Lancellotti P, Muraru D, Picard MH, Rietzschel ER, Rudski L, Spencer KT, Tsang W, Voigt JU : Recommendations for cardiac chamber quantification by echocardiography in adults : an update from the American Society of Echocardiography and the European Association of Cardiovascular Imaging. J Am Soc Echocardiogr 28 : 1-39, 2015

9. Cornette J, Laker S, Jeffery B, Lombaard H, Alberts A, Rizopoulos D, Roos-Hesselink JW, Pattinson RC : Validation of maternal cardiac output assessed by transthoracic echocardiography against pulmonary artery catheterization in severely ill pregnant women:prospective comparative study and systematic review. Ultrasound Obstet Gynecol 49 : 25-31, 2017

10. Bernstein DP, Lemmens HJ : Stroke volume equation for impedance cardiography. Med Biol Eng Comput 43 : 443450, 2005

11. Schmidt C, Theilmeier G, Van Aken H, Korsmeier P, Wirtz SP, Berendes E, Hoffmeier A, Meissner A : Comparison of electrical velocimetry and transoesophageal Doppler echocardiography for measuring stroke volume and cardiac output. Br J Anaesth 95 : 603-610, 2005

12. Zoremba N, Bickenbach J, Krauss B, Rossaint R, Kuhlen R, Schälte G : Comparison of electrical velocimetry and thermodilution techniques for the measurement of cardiac output. ActaAnaesthesiolScand 51 : 1314-1319, 2007

13. Archer TL, Conrad BE, Suresh P, Tarsa M : Electrical velocimetry demonstrates the increase in cardiac output and decrease in systemic vascular resistance accompanying cesarean delivery and oxytocin administration. J Clin Anesth 24 : 79-82,
2012

14. Liu Y, Pian-Smith MC, Leffert LR, Minehart RD, Torri A, Coté C, Kacmarek RM, Jiang Y : Continuous measurement of cardiac output with the electrical velocimetry method in patients under spinal anesthesia for cesarean delivery. J Clin Monit Comput 29 : 627-634, 2015

15. Grollmuss O, Gonzalez P : Non-invasive cardiac output measurement in low and very low birth weight infants : a method comparison. Front Pediatr $2: 16,2014$

16. Ho JG, Cohen MD, Ebenroth ES, Schamberger MS, Cordes TM, Bramlet MT, Hurwitz RA, Johnson TR: Comparison between transthoracic echocardiography and cardiac magnetic resonance imaging in patients status post atrial switch procedure. Congenit Heart Dis 7 : 122-130, 2012

17. Meßner NM, Zöllner FG, Kalayciyan R, Schad LR: Preclinical functional magnetic resonance imaging part II : the heart. Z Med Phys $24:$ 307-322, 2014

18. Agarwal R, Sola S : Cardiovascular MRI : what cardiologists need to know. Indian Heart J 59 : 69-76, 2007

19. Robson SC, Dunlop W, Boys RJ, Hunter S : Cardiac output during labour. Br Med J (Clin Res Ed) 295 : 1169-1172,1987

20. San-Frutos L, Engels V, Zapardiel I, Perez-Medina T, AlmagroMartinez J, Fernandez R, Bajo-Arenas JM : Hemodynamic changes during pregnancy and postpartum : a prospective study using thoracic electrical bioimpedance.J Matern Fetal Neonatal Med 24 : 1333-1340, 2011

21. Kamiya CA, Kitakaze M, Ishibashi-Ueda H, Nakatani S, Murohara T, Tomoike H, Ikeda T : Different characteristics of peripartum cardiomyopathy between patients complicated with and without hypertensive disorders : results from the Japanese nationwide survey of peripartum cardiomyopathy. Circ J 75 : 1975-1981, 2011

22. Ruys TP, Roos-Hesselink JW, Hall R, Subirana-Domènech MT, Grando-TingJ, Estensen M, Crepaz R, Fesslova V, Gurvitz M, De Backer J, Johnson MR, Pieper PG : Heart failure in pregnant women with cardiac disease:data from the ROPAC. Heart $100: 231-238,2014$

23. Norozi K, Beck C, Osthaus WA, Wille I, Wessel A, Bertram H : Electrical velocimetry for measuring cardiac output in children with congenital heart disease. Br J Anaesth 100 : 88-94,2008

24. Phillips R, Lichtenthal P, Sloniger J, Burstow D, West M, Copeland J : Noninvasive cardiac output measurement in heart failure subjects on circulatory support. Anesth Analg $108: 881-886,2009$

25. Marqué S, Cariou A, Chiche JD, Squara $\mathrm{P}$ : Comparison between Flotrac-Vigileo and Bioreactance, a totally noninvasive method for cardiac output monitoring. Crit Care 13 : R73, 2009

26. Squara P, Denjean D, Estagnasie P, Brusset A, Dib JC, Dubois C : Noninvasive cardiac output monitoring (NICOM) : a clinical validation. Intensive Care Med 33 : 1191-1194,2007 